\title{
Cutaneous Protothecosis
}

\author{
Dana M. Baigrie, DO; Laura F. Sandoval, DO; Warren J. White, MD; Laura Tanner, MD; \\ Warren C. White, MD
}

\section{PRACTICE POINTS}

- Cutaneous protothecosis is a rare skin infection most commonly reported in immunocompromised individuals with recent exposure to contaminated soil or water. Cutaneous protothecosis has the potential to mimic many other skin diseases and lesions, including eczema; nonmelanoma skin cancer; or bacterial, viral, and fungal skin infections.

- A skin biopsy is essential for diagnosis, and histopathology is characteristic with soccer ball-appearing morula noted in a mixed inflammatory infiltrate.

To the Editor:

Protothecosis infections are caused by an achlorophyllic algae of the species Prototheca. Prototheca organisms are found mostly in soil and water. ${ }^{1}$ Human infections are rare and involve 2 species, Prototheca wickerhamii and Prototheca zopfii. The former most commonly is responsible for human infections, though $P$ zopfii results in more serious systemic infections with a poor prognosis. There are various types of Prototheca infection presentations, with a 2007 review of 117 cases reporting that cutaneous infections are most common (66\%), followed by systemic infections (19\%), and olecranon bursitis $(15 \%){ }^{2}$ Skin lesions most commonly occur on the extremities and face, and they present as vesiculobullous and ulcerative lesions with purulent drainage. The skin lesions also may appear as erythematous plaques or nodules, subcutaneous papules, verrucous or herpetiformis lesions, or pyogenic granuloma-like lesions. ${ }^{3}$ Protothecosis typically affects immunocompromised individuals, especially those with a history of chronic corticosteroid use, malignancy, diabetes mellitus, AIDS, and/or organ transplant. ${ }^{1}$ We present a case of cutaneous protothecosis on the dorsal distal extremity of a 94-year-old woman. History of exposure to soil while gardening was elicited from the patient, and no immunosuppressive history was present aside from the patient's age. This case may prompt workup for malignancy or immunosuppression in this patient subset.

A 94-year-old woman with a medical history of cutaneous squamous cell carcinoma (SCC) presented with a growing lesion on the dorsal surface of the left fourth digit of 2 months' duration. The patient reported the lesion was painful, and she noted preceding trauma to the area that was suspected to have occurred while gardening. Physical examination revealed an ulcerated, hypertrophic, erythematous nodule on the dorsal surface of the left fourth metacarpophalangeal joint. The differential diagnosis included SCC, inflamed cyst, verruca vulgaris, and orf virus due to the clinical presentation. A shave biopsy was performed, and the lesion subsequently was treated with electrodesiccation and curettage.

Histopathologic evaluation revealed pseudoepitheliomatous hyperplasia with a mixed inflammatory infiltrate including lymphocytes and histiocytes. A morula within the dermis was characteristic of a protothecosis infection (Figure 1). On follow-up visit 6 weeks later, the lesion had grown back to its original size and morphology (Figure 2). At this time, the lesion was again treated with shave removal, followed by electrodesiccation and curettage, and the patient was placed on oral fluconazole $200 \mathrm{mg}$ daily for 1 month. When the lesion did not resolve with fluconazole, she was referred to infectious disease as

Drs. Baigrie and Sandoval are from the Department of Dermatology, Sampson Regional Medical Center, Clinton, North Carolina, and Campbell University, Buies Creek, North Carolina. Drs. WJ White and WC White are from Coastal Carolina Pathology, Wilmington, North Carolina. Dr. Tanner is from the Department of Dermatology, Wilmington Health, North Carolina.

The authors report no conflict of interest.

Correspondence: Dana M. Baigrie, DO, 1099 Medical Center Dr, \#201, Wilmington, NC 28401 (dbaigrie@vcom.edu).

doi: $10.12788 /$ cutis. 0334 
FIGURE 1. A, Histopathology revealed pseudoepitheliomatous hyperplasia with a mixed inflammatory infiltrate including lymphocytes and histiocytes $(H \& E$, original magnification $\times 10$ ) $B$, Within the dermis was the characteristic morula representing a protothecosis infection (H\&E, original magnification $\times 40$ ).
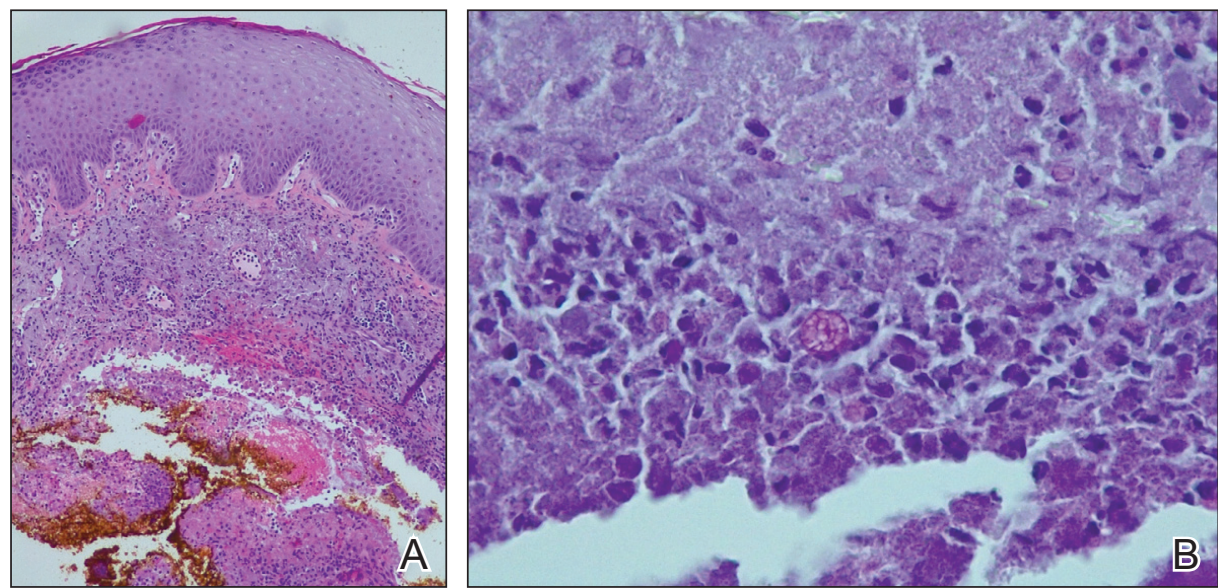

Cutaneous protothecosis has the potential to mimic many other skin diseases and lesions, and, given its rarity, it may not be on the radar of dermatologists. Our patient's lesion was presumed to be a skin cancer and was treated as such because of the history of SCC and clinical presentation. Although excision of individual lesions of protothecosis can be curative, electrodesiccation and curettage does not appear to be an adequate treatment, as the lesion subsequently recurred. It also is possible that this case represents $P$ zopfii infection, as it did not respond to treatment with oral fluconazole, though in vitro studies with fluconazole to both $P$ zopfii and $P$ wickerhamii had variable treatment success. ${ }^{2}$ Also, the histopathologic findings were most consistent with $P$ wickerhamii, revealing small, round, symmetrical morula, compared to $P$ zopfii, which typically will display oval or cylindrical, asymmetrical, random internal segmentation. ${ }^{5}$ This case may warrant determination of species, which can be accomplished by a culture on Sabouraud dextrose agar, carbohydrate and alcohol assimilation test, yeast biochemical card, serological typing by immunoblotting, immunofluorescence study using speciesspecific antibodies, or amplification by polymerase chain reaction for small subunit ribosomal DNA sequences. ${ }^{2,6-8}$

The natural history of isolated skin disease is an indolent progressive course; however, reports do exist noting spontaneous resolution. ${ }^{4,9}$ Treatment options for Prototheca infections can be disappointing and consist of both surgical and medical management, or a combination of the 2 approaches. Reports in the literature support the use of antifungals including ketoconazole, voriconazole, itraconazole, fluconazole, and amphotericin B, with the latter displaying the best activity against Prototheca species. ${ }^{2}$ Tetracycline has been used in combination with oral or topical amphotericin B and was found to be synergistic in vitro and in case reports at successfully treating cutaneous protothecosis infections. It is possible that our patient was not treated with fluconazole long enough for it to become therapeutic, as most reported treatment regimens are weeks to months in length. Conversely, it 
may have been of benefit to transition the patient to topical amphotericin B and tetracycline, as fluconazole failed in this patient. However, treatment successes and failures are limited to case reports/case series and in vitro studies, with prospective studies lacking. Due to the variability with in vitro susceptibility profiles for Prototheca species, it generally is not recommended to pursue in vitro susceptibility testing in the management of Prototheca skin infections due to the inconsistency demonstrated between in vitro activity and clinical response to therapy. ${ }^{2}$

\section{REFERENCES}

1. Silva PC, Costa e Silva SB, Lima RB, et al. Cutaneous protothecosiscase report. An Bras Dermatol. 2013;88:183-185.

2. Lass-Flörl C, Mayr A. Human protothecosis. Clin Microbiol Rev. 2007;20:230-242
3. Seok JY, LeeY, Lee H, et al. Human cutaneous protothecosis: report of a case and literature review. Korean J Pathol. 2013;47:575-578.

4. Mayorga J, Barba-Gómez JF, Verduzco-Martínez AP, et al. Protothecosis. Clin Dermatol. 2012;30:432-436.

5. Walsh SV, Johnson RA, Tahan SR. Protothecosis: an unusual cause of chronic subcutaneous and soft tissue infection. Am J Dermatopathol. 1998;20:379-382.

6. Casal MJ, Gutierrez J. Simple new test for rapid differentiation of Prototheca wickerhamii from Prototheca zopfii. J Clin Microbiol. 1983;18:992-993.

7. Arnold, P, Ahearn, DG. The systematics of the genus Prototheca with a description of a new species P. filamenta. Mycologia 1972;64:265-275.

8. Roesler U, Scholz H, Hensel H. Emended phenotypic characterization of Prototheca zopfii: a proposal for three biotypes and standards for their identification. Int J Syst Evol Microbiol. 2003;53:1195-1199.

9. Todd JR, King JW, Oberle A, et al. Protothecosis: report of a case with 20-year follow-up, and review of previously published cases. Med Mycol. 2012;50:673-689.

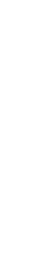

